\title{
Protective activity of kudzu (Pueraria thunbergiana) vine on chemically-induced hepatotoxicity: in vitro and in vivo studies
}

\author{
Bo Yoon Chang ${ }^{1}$, Dong-Sung Lee ${ }^{2}$, Jun-Kyoung Lee ${ }^{3}$, Youn-Chul Kim ${ }^{1}$, Hyoung-Kwon Cho ${ }^{3}$ and Sung Yeon Kim*
}

\begin{abstract}
Background: Kudzu (Pueraria thunbergiana) root has long been used in Traditional Chinese Medicine. However, the vine of the kudzu plant has been considered waste material. This study aimed to investigate the hepatoprotective properties of the kudzu vine.

Methods: We created 0\%, $30 \%, 70 \%$, and $95 \%$ ethanolic kudzu vine extracts. The isoflavone contents of kudzu vine extract were quantified by high-performance liquid chromatography. Tertiary-butylhydroperoxide (t-BHP) was added to human liver-derived HepG2 cells, and the production of reactive oxygen species was measured in the presence and absence of kudzu vine extract. Antioxidant activity was evaluated in all kudzu vine extracts using a hydroxyradical scavenging assay. Thirty-five male Sprague-Dawley rats were divided into seven groups $(n=5)$; two groups were not given any extract or drug, one group was treated with $50 \mathrm{mg} / \mathrm{kg}$ silymarin orally for 5 days, and the remaining four groups were respectively treated with $100 \mathrm{mg} / \mathrm{kg}$ of $0 \%, 30 \%, 70 \%$, or $95 \%$ ethanolic extract of kudzu vine orally once daily for 5 days. On day 5 the treatment groups and one untreated group were fed 0.75 $\mathrm{ml} / \mathrm{kg}$ carbon tetrachloride $\left(\mathrm{CCl}_{4}\right)$ to induce liver damage. Blood and liver tissue samples were collected $24 \mathrm{~h}$ after $\mathrm{CCl}_{4}$ administration for measurement of plasma alanine aminotransferase and aspartate aminotransferase, and concentration of malondialdehyde and glutathione in liver tissue.

Results: Puerarin was the most abundant isoflavone in kudzu vine extract. Kudzu vine extract significantly reduced the cytotoxicity and production of reactive oxygen species induced by $t$-BHP in a dose-dependent manner. Treatment with $0 \%$ and $30 \%$ ethanolic extracts of kudzu vine significantly lowered the plasma levels of alanine aminotransferase and aspartate aminotransferase in a $\mathrm{CCl}_{4}$-induced hepatotoxicity rat model $(P<0.05)$. Glutathione was significantly elevated in the $30 \%$ ethanolic extract-treated group $(P<0.05)$, while the malondialdehyde level in liver tissue was significantly decreased in the $0 \%$ and $30 \%$ ethanolic extract-treated groups $(P<0.05)$.
\end{abstract}

Conclusions: The kudzu vine is potentially highly beneficial in treating liver damage, as it scavenges reactive free radicals and boosts the endogenous antioxidant system.

Keywords: Kudzu vine, Anti-oxidant, Hepatoprotection, $t-\mathrm{BHP}, \mathrm{CCl}_{4}$

\footnotetext{
* Correspondence: sungykim@wonkwang.ac.kr

${ }^{1}$ Institute of Pharmaceutical Research and Development, College of

Pharmacy, Wonkwang University, Shinyoung-dong 344-2, Iksan, Jeonbuk

570-749, Republic of Korea

Full list of author information is available at the end of the article
} 


\section{Background}

Kudzu (Pueraria thunbergiana) is native to Korea, Japan, China, and India [1], and is now grown worldwide. Kudzu root is used as a source of starch in China and Japan, and is also eaten as a vegetable [2]; it has been used in Traditional Chinese Medicine to treat conditions including the common cold, headache, diarrhoea and heart disease, and is also purported to have various other beneficial effects. Puerarin (4',7-dihydroxy-8- $\beta$-D-glucosylisoflavone) is the major isoflavone compound isolated from kudzu [3, 4]; it has a variety of biological actions in cardiovascular disease, gynaecological disease, osteoporosis, cognitive capability and diabetic nephropathy, including antioxidant activity [3-7].

In contrast to the kudzu root, the kudzu vine is considered to be a waste product with limited usage. The kudzu vine grows one foot per day, outcompeting all other vegetation and making it one of the most noxious invasive plant species in the USA $[8,9]$. Kudzu has had the greatest deleterious impact on the forestry industry, where annual productivity losses have been estimated at 100-500 million USD [9].

Recently, kudzu vine has begun to be considered as a new resource. There are currently only few papers investigating the effects of the kudzu vine, including one which reported that kudzu vine effectively reduced bone loss in ovariectomized mice [7]. The kudzu vine contains isoflavones in a similar ratio to those of the kudzu root; hence, the kudzu vine could be a valuable new source of polyphenolic compounds, including isoflavones, which can play an important role as dietary antioxidants [10]. Antioxidants terminate chain reactions by removing free radical intermediates, and inhibit other oxidation reactions by being oxidized themselves. Oxidative intermediates exert their toxic effect through destruction of cellular defence mechanisms $[10,11]$. Oxidative stress is a key phenomenon in chronic diseases and hepatotoxicity induced by various chemicals [11]. Until now the hepatoprotective effect of kudzu vine has not been investigated.

The present study was designed to evaluate the effects of kudzu vine extract on hepatic injuries caused by the oxidative stressors tertiary-butylhydroperoxide ( $t$-BHP) and carbon tetrachloride $\left(\mathrm{CCl}_{4}\right)$. $t$-BHP has often been used in models to screen the hepatoprotective activity of drugs to investigate the mechanism of cell injury initiated by acute oxidative stress [12], while $\mathrm{CCl}_{4}$ is used as a model for studying free radical-induced liver injury and screening hepatoprotective drugs $[12,13]$.

\section{Methods}

\section{Preparation of kudzu vine extract}

The kudzu vine was collected from Jinan Province, Jeonbuk, Korea in November 2010. The botanical identification was authenticated by Dr Hyoung-Kwon Cho of the Han Poong Pharmaceutical Co., Ltd, where the voucher specimen (\#FPR-36) was deposited.

Dried and pulverized kudzu vines (2 kg) were boiled with $2 \mathrm{~L}$ of distilled water and various concentrations of ethanol for $3 \mathrm{~h}$. The extracts were concentrated at reduced pressure in a rotary evaporator (N-1000S, EYELA, Japan), yielding a water extract (439.5 g), $30 \%$ ethanolic extract (409.2 g), $70 \%$ ethanolic extract $(436.7 \mathrm{~g})$, and a $95 \%$ ethanolic extract (284.8 g).

High-performance liquid chromatography (HPLC) analysis The isoflavone contents of the kudzu vine extracts were quantified by HPLC (Waters 2695 system, Waters Co., Milford, MA, USA) using a CAPCELLPAK UG 120 $250 \times 4.6 \mathrm{~mm}, 5 \mu \mathrm{m}$ (Shiseido, Tokyo, Japan). Separation and quantification were achieved at $30{ }^{\circ} \mathrm{C}$ using a gradient from solution A (a $10 \%$ aqueous methanol solution with $2 \%$ acetic acid) to solution B (a $98 \%$ aqueous methanol solution with $2 \%$ acetic acid) at a flow rate of $1 \mathrm{ml} / \mathrm{min}$. Peaks were detected at $260 \mathrm{~nm}$. The presence of isoflavones was confirmed by observing a retention time that was the same as that of appropriate standards (Wako Chemical Co., Osaka, Japan).

\section{t-BHP-induced hepatotoxicity in vitro model Cell culture and viability assay}

Human liver-derived HepG2 cells were obtained from American Type Culture Collection (Manassas, VA). The monolayer HepG2 cell culture was trypsinized and the cell count was adjusted to $1.0 \times 10^{5}$ cells $/ \mathrm{ml}$ using Dulbecco's Modified Eagle Medium containing $10 \%$ foetal bovine serum. $0.1 \mathrm{ml}$ of the diluted cell suspension was added to each well of the 96-well microplate. After $24 \mathrm{~h}$ a partial monolayer had formed; the supernatant was removed and the monolayer was washed once with medium. Cultures were treated with $t$-BHP in the presence or absence of kudzu vine extract and incubated for $12 \mathrm{~h}$. Curcumin $(20 \mu \mathrm{M})$ was used as a reference control. For determination of cell viability, $50 \mathrm{mg} / \mathrm{ml} 3$-[4,5-dimethylthiazol-2-yl]-2,5-diphenyltetrazolium bromide was added to $1 \mathrm{ml}$ of cell suspension $\left(1 \times 10^{5}\right.$ cells $\mathrm{per} / \mathrm{ml}$ in 96-well plates) for $4 \mathrm{~h}$, and the formazan formed was dissolved in acidic 2-propanol. Optical density was measured at $590 \mathrm{~nm}$.

\section{Measurement of reactive oxygen species (ROS)}

HepG2 cells $\left(2.5 \times 10^{4}\right.$ cells $/ \mathrm{ml}$ per $1 \mathrm{ml}$ in 24 -well plates $)$ were treated with $100 \mu \mathrm{M}$ of $t$-BHP in the presence or absence of the kudzu vine extract and incubated for 12 h. After washing with phosphate-buffered saline (PBS), the cells were stained with $10 \mu \mathrm{M}$ 20,70-dichlorofluorescein diacetate in Hank's balanced salt solution for 30 min in the dark. The cells were then washed twice with 
PBS and extracted with $1 \%$ Triton X-100 in PBS at $37{ }^{\circ} \mathrm{C}$ for $10 \mathrm{~min}$. Fluorescence was measured at an excitation wavelength of $490 \mathrm{~nm}$ and an emission wavelength of $525 \mathrm{~nm}$ (Spectramax Gemini XS; Molecular Devices, Sunnyvale, CA, USA). Curcumin $(20 \mu \mathrm{M})$ was used as a reference control.

\section{Hydroxyl radical scavenging assay}

Hydroxyl radical scavenging assay was performed using the method described by Klouwen [14]. The reaction solution was incubated at $37{ }^{\circ} \mathrm{C}$ for $30 \mathrm{~min}$. Absorbance was measured at $520 \mathrm{~nm}$ using a UV-Vis spectrophotometer (Spectramax Gemini XS; Molecular Devices, Sunnyvale, CA, USA). The inhibition rate was calculated as follows: $\left[\left(A_{1}-A_{2}\right) /\left(A_{0}-A_{2}\right)\right] \times 100 \%$, where $A_{0}$ is the absorbance of the control, $A_{1}$ is the absorbance of the sample, and $A_{2}$ is the absorbance of a blank sample. Vitamin $C(50 \mu \mathrm{M})$ was used as a reference control.

\section{$\mathrm{CCl}_{4}$-induced hepatotoxicity in vivo model Experimental animals and design}

Thirty-five male Sprague-Dawley rats (weighing 200-220 g) supplied by Hanil Laboratory Animal Research (Pyeongtaek, Korea) were fed a standard diet (Orient Bio, Korea) and provided with tap water ad libitum. This study was approved by the Wonkwang University Animal Care Committee.
The rats were divided equally into seven groups $(n=5$ in each group); two groups were not treated with any extract or drug, while the respective treatment groups were fed either $0 \%, 30 \%, 70 \%$, or $95 \%$ kudzu vine extract $(100 \mathrm{mg} / \mathrm{kg})$ or the reference drug silymarin (50 $\mathrm{mg} / \mathrm{kg}$ ) for 5 consecutive days. Acute liver injury was induced by a single administration of oral $0.75 \mathrm{ml} / \mathrm{kg} \mathrm{CCl}_{4}$ diluted in corn oil $1 \mathrm{~h}$ to rats in all treatment groups after the final dose of kudzu vine extract or silymarin was administered, and to one of the untreated groups (Fig. 1). The rats were euthanized $24 \mathrm{~h}$ later by exsanguination from the abdominal aorta under ether-induced anaesthesia, then blood and liver tissue samples were collected.

\section{Estimation of biochemical parameters}

The separated plasma was used for the determination of plasma levels of aspartate aminotransferase (AST) and alanine aminotransferase (ALT); the enzyme level was measured according to the method described by Reitman and Frankel [15]. To determine the level of malondialdehyde (MDA), the end product of lipid peroxidation was measured using a thiobarbituric acid reactive substance assay with some modification [16]; the liver was pulverized using a polytron, and then the homogenate was estimated and the supernatant was separated. The total glutathione

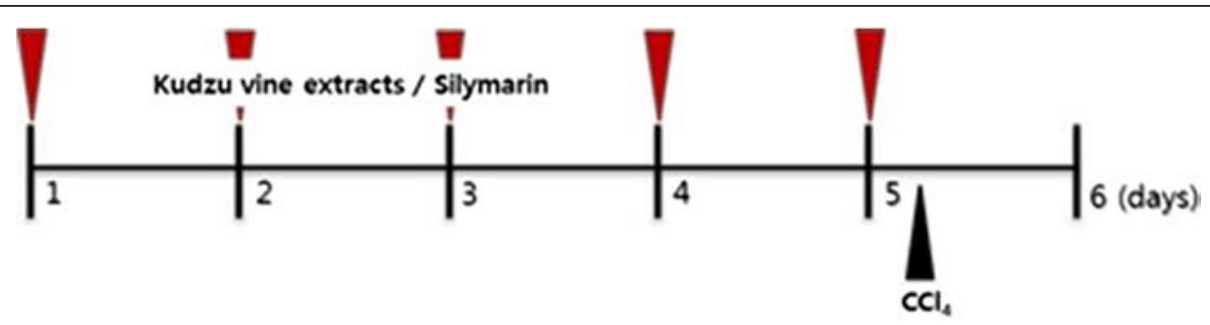

\begin{tabular}{|c|c|c|}
\hline NO. & & Group \\
\hline 1 & Normal & \\
\hline 2 & $\mathrm{CCl}_{4}$ only & \\
\hline 3 & \multirow{4}{*}{$\begin{array}{c}\text { Kudzu vine } \\
(100 \mathrm{mg} / \mathrm{kg}) \\
+ \\
\mathrm{CCl}_{4} \\
(0.75 \mathrm{~mL} / \mathrm{kg})\end{array}$} & $0 \% \mathrm{EtOH}$ \\
\hline 4 & & $30 \% \mathrm{EtOH}$ \\
\hline 5 & & $70 \%$ EtOH \\
\hline 6 & & 95\% EtOH \\
\hline 7 & \multicolumn{2}{|c|}{ Reference control: silymarin $(50 \mathrm{mg} / \mathrm{kg})$} \\
\hline
\end{tabular}

Fig. $1 \mathrm{CCl}_{4}$-induced hepatotoxicity in vivo study design. The rats were divided into the following seven groups, with five animals in each group 
(GSH) was measured according to the method described by Griffith et al. [17].

\section{Statistical analysis}

Data were expressed as mean \pm SD. Significant differences were compared using repeated measures ANOVA followed by the Newman-Keuls multiple range test. Statistical significance was defined as $P<0.05$. All statistical analyses were performed using SPSS v. 20.0 (IBM, Armonk, NY, USA).

\section{Results}

\section{Isoflavone composition of kudzu vine extract}

Isoflavone contents of kudzu vine extract were quantitated; the isoflavone aglycone and glycoside concentrations in each extract are listed in Table 1. Puerarin was the most abundant isoflavone in kudzu vine extract (Fig. 2).

\section{Effects of kudzu vine extract on $t$-BHP-induced hepatotoxicity and free radical scavenging activity}

Treatment with $t$-BHP for $12 \mathrm{~h}$ increased HepG2 cell death by 2.2 -fold compared with untreated cells, while the addition of kudzu vine extracts at non-cytotoxic concentrations $(12.5,25,50$, and $100 \mu \mathrm{g} / \mathrm{ml})$ caused a significant dose-dependent elevation in cell viability (Fig. 3a).

ROS generation was increased 2.3 -fold by $t$-BHP stimulation of hepatocytes. The addition of kudzu vine extracts caused a significant dose-dependent reduction in ROS generation (Fig. 3b). Curcumin was used as a reference control, and treatment with curcumin exhibited significant cytoprotective effects and ROSscavenging activity [18].

The antioxidant activity of the kudzu vine extracts was compared with a representative antioxidant, vitamin $\mathrm{C}$, by hydroxyradical scavenging assay. The kudzu vine extracts at concentrations of 10,30 , and $100 \mu \mathrm{g} / \mathrm{ml}$ exhibited concentration-dependent radical scavenging reactions, from 5.41 to $31.8 \%$ inhibition. The antioxidant activity of $100 \mu \mathrm{g} / \mathrm{ml}$ kudzu vine extract was similar to that of vitamin C (Fig. 4).
Effect of the kudzu vine extract on markers of hepatic damage

The hepatoprotective effects of the kudzu vine extract are summarized in Table 2. Rats treated with a single dose of $\mathrm{CCl}_{4}$ developed hepatic damage. Compared with the normal group, the $\mathrm{CCl}_{4}$-treated rats showed a 3 -fold increase in plasma ALT $(140.0 \pm 4.4 \mathrm{unit} / \mathrm{ml})$ and AST (167.3 \pm $10.0 \mathrm{unit} / \mathrm{ml})$. The $0 \%$ and $30 \%$ ethanolic extracts significantly blocked the $\mathrm{CCl}_{4}$-induced elevation of plasma ALT (106 \pm 8.9 and $97.3 \pm 4.5$ units $/ \mathrm{ml}$ respectively) and AST (128.8 \pm 5.0 and $99.5 \pm 4.1$ units $/ \mathrm{ml}$ respectively); similar effects were observed in the silymarin-treated group.

\section{Effect of the kudzu vine extract on hepatic antioxidant markers}

The amount of MDA increased about 4-fold in the $\mathrm{CCl}_{4}$-treated group $(58 \pm 5.6 \mathrm{nmol} / \mathrm{g}$ liver $)$ compared with the normal group. However, the $0 \%$ and $30 \%$ ethanolic extracts both significantly inhibited cell damage (MDA $16.0 \pm 1.57$ and $32.3 \pm 3.0 \mathrm{nmol} / \mathrm{g}$ liver respectively; Fig. 5a).

The $\mathrm{CCl}_{4}$-treated group had significantly reduced $\mathrm{GSH}$ levels $(5.74 \pm 0.1 \mathrm{nmol} / \mathrm{g}$ liver) compared with the normal group (7.31 $\pm 0.4 \mathrm{nmol} / \mathrm{g}$ liver). The $30 \%$ ethanolic kudzu vine extract elicited a significant increase in GSH levels $(6.17 \pm 0.06 \mathrm{nmol} / \mathrm{g}$ liver $)$ compared with the GSH levels of the $\mathrm{CCl}_{4}$-treated group (Fig. 5b).

\section{Discussion}

The present study found that puerarin was the most abundant isoflavone in kudzu vine extract. In vitro experiments on human liver-derived HepG2 cells showed that kudzu vine extract had cytoprotective and antioxidant properties. In vivo experiments on rats showed that $0 \%$ and $30 \%$ kudzu vine ethanolic extracts significantly reduced hepatic damage (as measured by increase in ALT and AST) and hepatic lipid peroxidation (as measured by increase in MDA), and $30 \%$ kudzu vine extract caused a significant increase in the antioxidant GSH.

Liver disease remains one of the most serious health problems worldwide. The liver is the main organ that metabolizes xenobiotics to help eliminate waste from the body; hence, the liver is exposed to a high concentration of chemicals, drugs, and natural products, which can lead to liver dysfunction, cell injury, and organ failure

Table 1 Composition of isoflavones in kudzu vine extract

\begin{tabular}{lccccccc}
\hline & Puerarin $(\mathrm{mg} / \mathrm{g})$ & Daidzin $(\mathrm{mg} / \mathrm{g})$ & Glycitin $(\mathrm{mg} / \mathrm{g})$ & Genistin $(\mathrm{mg} / \mathrm{g})$ & Daidzein $(\mathrm{mg} / \mathrm{g})$ & Glycitein $(\mathrm{mg} / \mathrm{g})$ & Genistein $(\mathrm{mg} / \mathrm{g})$ \\
\hline $\mathrm{H}_{2} \mathrm{O}$ & 236.5 & 3.33 & 3.45 & 3.33 & 6.27 & 0.27 & 0.29 \\
$30 \% \mathrm{EtOH}$ & 303.3 & 4.33 & 3.83 & 4.33 & 9.22 & 0.39 & 0.60 \\
$70 \% \mathrm{EtOH}$ & 288.1 & 4.26 & 3.90 & 4.26 & 10.1 & 0.33 & 0.71 \\
$95 \% \mathrm{EtOH}$ & 372.8 & 4.50 & 4.52 & 4.50 & 14.9 & 0.56 & 1.00 \\
\hline
\end{tabular}

$\mathrm{EtOH}$ ethanol 


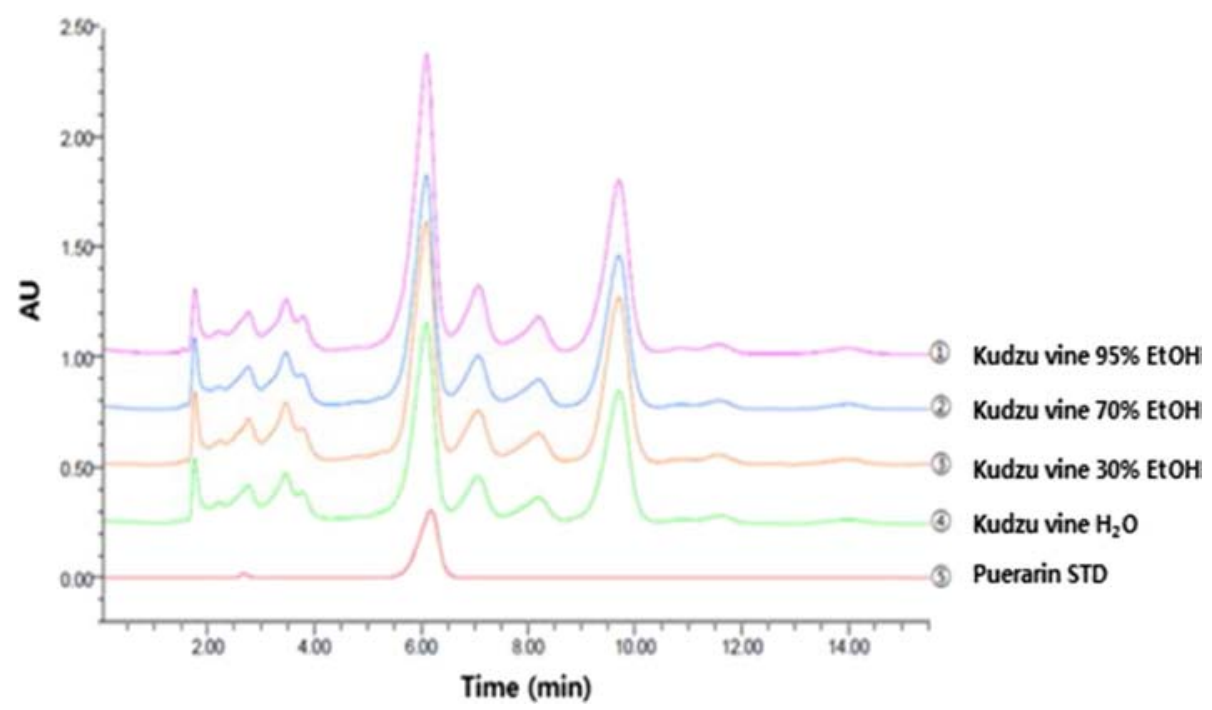

Fig. 2 High performance liquid chromatogram of puerarin in crude extract from kudzu vine at $260 \mathrm{~nm}$

A

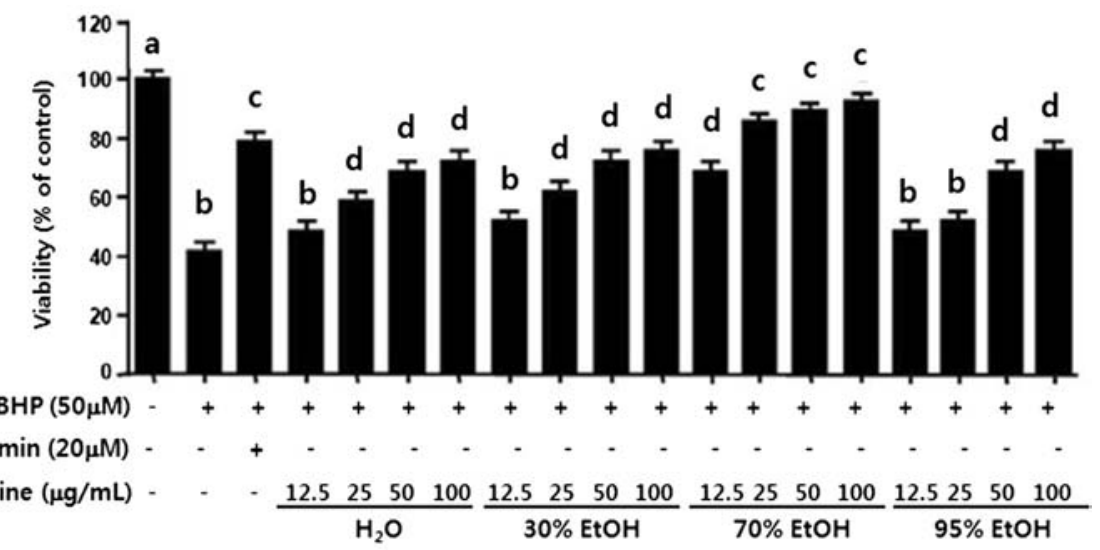

B

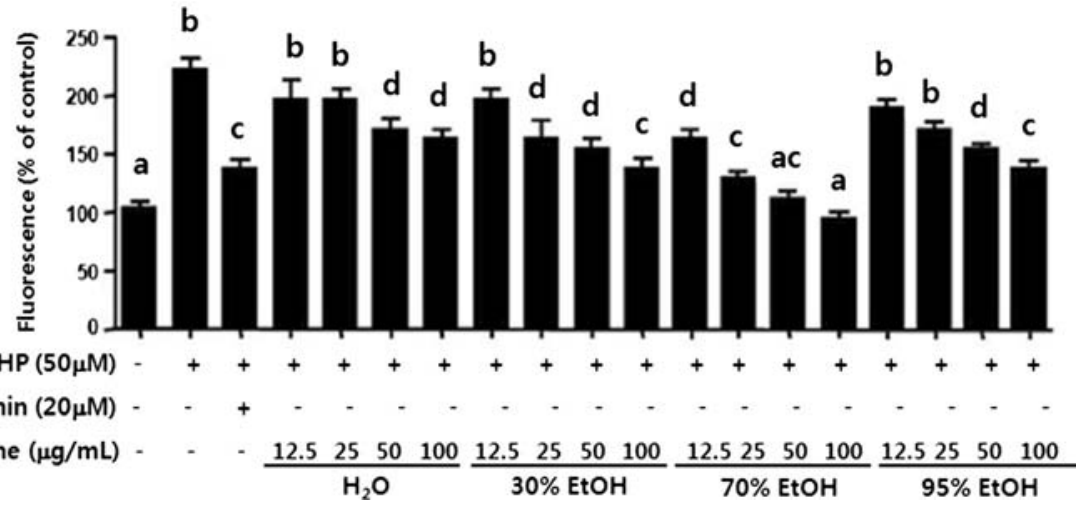

Fig. 3 Effects of kudzu vine extract on t-BHP-induced (a) hepatotoxicity and (b) inhibition of ROS generation. The effects of kudzu vine extract on hepatotoxicity and inhibition of ROS generation in t-BHP-treated human liver-derived HepG2 cells were investigated. Curcumin was used as a reference control. The results are presented as mean \pm SD of three experiments with triplicate samples. Values with different letters $(a, b, c, d)$ are significantly different one from another (one-way ANOVA followed by Newman-Keuls multiple range test, $P<0.05$ ). 


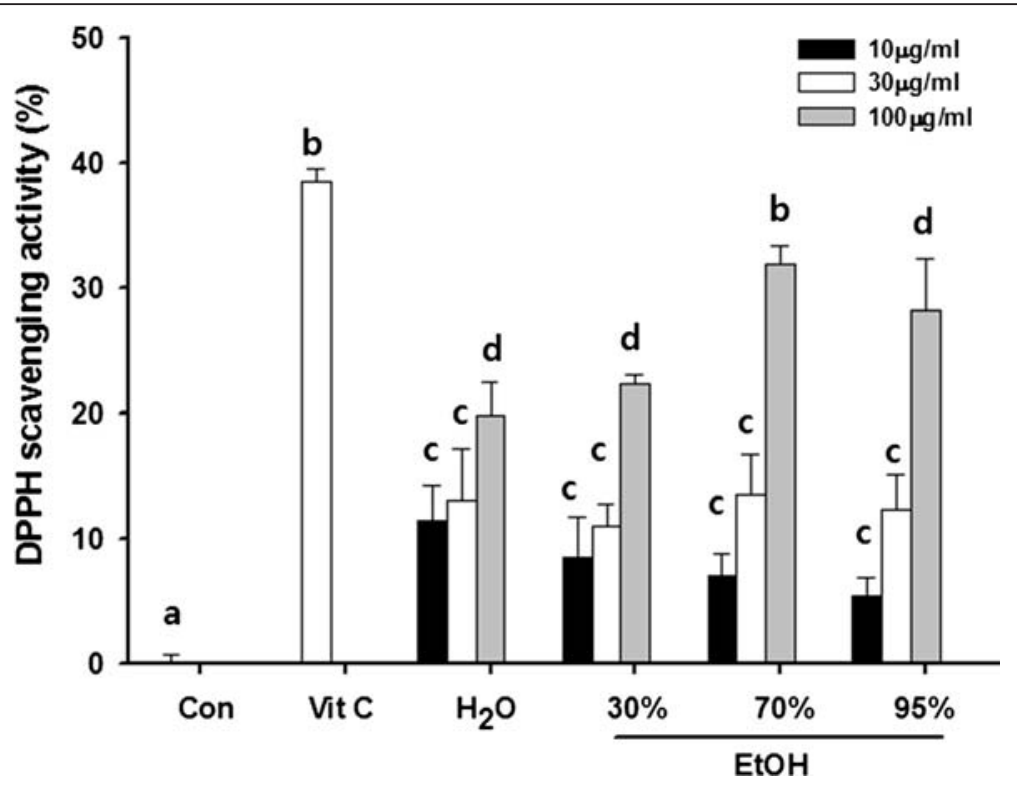

Fig. 4 Effect of kudzu vine extract on radical scavenging activity. Vitamin C was used as a reference control. The results are presented as mean \pm SD. Values with different letters $(a, b, c, d)$ are significantly different one from another (one-way ANOVA followed by Newman-Keuls multiple range test, $P<0.05)$

[19]. More than 100 human diseases, including liver diseases, are related to oxidative stress [20].

Herbal plants have recently gained attention as potential treatments for cancers, metabolic diseases, allergies, ischemia, and inflammation, and especially for their hepatoprotective activities. Most commonly, these hepatoprotective activities seem to be related to the antioxidant capacity of these plants. Naturally derived antioxidants counteract the oxidative stress induced by many hepatotoxins [21]. The quest to discover such naturally occurring antioxidants has become a major scientific focus over the past few decades. The antioxidant activities of isoflavones have been associated with the hepatoprotective effect of these compounds. Plant-derived isoflavone compounds are thought

Table 2 The effects of kudzu vine extracts on plasma alanine aminotransferase and aspartate aminotransferase levels in $\mathrm{CCl}_{4}$-treated rats

\begin{tabular}{llccc}
\hline Group & & Dose $(\mathrm{mg} / \mathrm{kg})$ & ALT (units/ml) & AST (units $/ \mathrm{ml})$ \\
\hline Normal & & & $35.4 \pm 5.2^{\mathrm{a}}$ & $60.9 \pm 3.2^{\mathrm{a}}$ \\
$\mathrm{CCl}_{4}$ only & & & $140.0 \pm 4.4^{\mathrm{b}}$ & $167.3 \pm 10.0^{\mathrm{b}}$ \\
Kudzu vine & $\mathrm{H}_{2} \mathrm{O}$ & 100 & $106.1 \pm 8.9^{\mathrm{c}}$ & $128.8 \pm 5.0^{\mathrm{c}}$ \\
$+\mathrm{CCl}_{4}$ & $30 \% \mathrm{EtOH}$ & 100 & $97.3 \pm 4.5^{\mathrm{c}}$ & $99.5 \pm 4.1^{\mathrm{c}}$ \\
& $70 \% \mathrm{EtOH}$ & 100 & $152.5 \pm 13.7^{\mathrm{b}}$ & $163.5 \pm 9.3^{\mathrm{b}}$ \\
& $95 \% \mathrm{EtOH}$ & 100 & $151.5 \pm 4.9^{\mathrm{b}}$ & $182.0 \pm 8.9^{\mathrm{b}}$ \\
Silymarin & & 50 & $100.4 \pm 3.8^{\mathrm{c}}$ & $95.5 \pm 4.9^{\mathrm{c}}$ \\
\hline
\end{tabular}

Results presented as mean \pm SD. Values with different letters $(\mathrm{a}, \mathrm{b}, \mathrm{c})$ are significantly different one from another (one-way ANOVA followed by Newman-Keuls multiple range test, $P<0.05$ ) to contribute to the prevention of diseases associated with oxidative stress [22].

Kudzu root is a rich source of isoflavone glucosides. Common isoflavones of kudzu root include puerarin (daidzein 8-C-glucoside), daidzin (daidzein 7-O-glucoside), daidzein, genistein and formononetin [7]. These isoflavones have been associated with antioxidant, hepatoprotective and other pharmacological effects [23, 24]. Among the isoflavones in kudzu root, puerarin is the most abundant (approximately $23 \% \mathrm{w} / \mathrm{w}$ ) and has attracted considerable attention because of its potent ability to cause various pharmacological effects [23]. A previous study found that puerarin was the most abundant isoflavone contained in the kudzu vine (nearly 50\%), followed by daidzin (3.58\%), daidzein $(0.92 \%)$, genistein $(0.03 \%)$ and other isoflavones $(0.5 \%)$ [7]; these results are consistent with the present study. Unlike kudzu root, the beneficial actions of kudzu vine against liver disease have not been extensively investigated.

In the present study, we investigated the antioxidant activity and the hepatoprotective effect of kudzu vine extract. $t$-BHP was used to induce hepatotoxicity in the HepG2 cell line. The cell viability and inhibition of ROS generation was then examined in vitro. All concentrations of kudzu vine extract significantly protected the cell from dying and inhibited the ROS production in a dose-dependent manner. There was a clear correlation between the ratio of protection and the level of antioxidant activity provided by kudzu treatment. ROS production from cells occurs via multiple mechanisms. Large numbers of oxygen free radicals are produced within the 

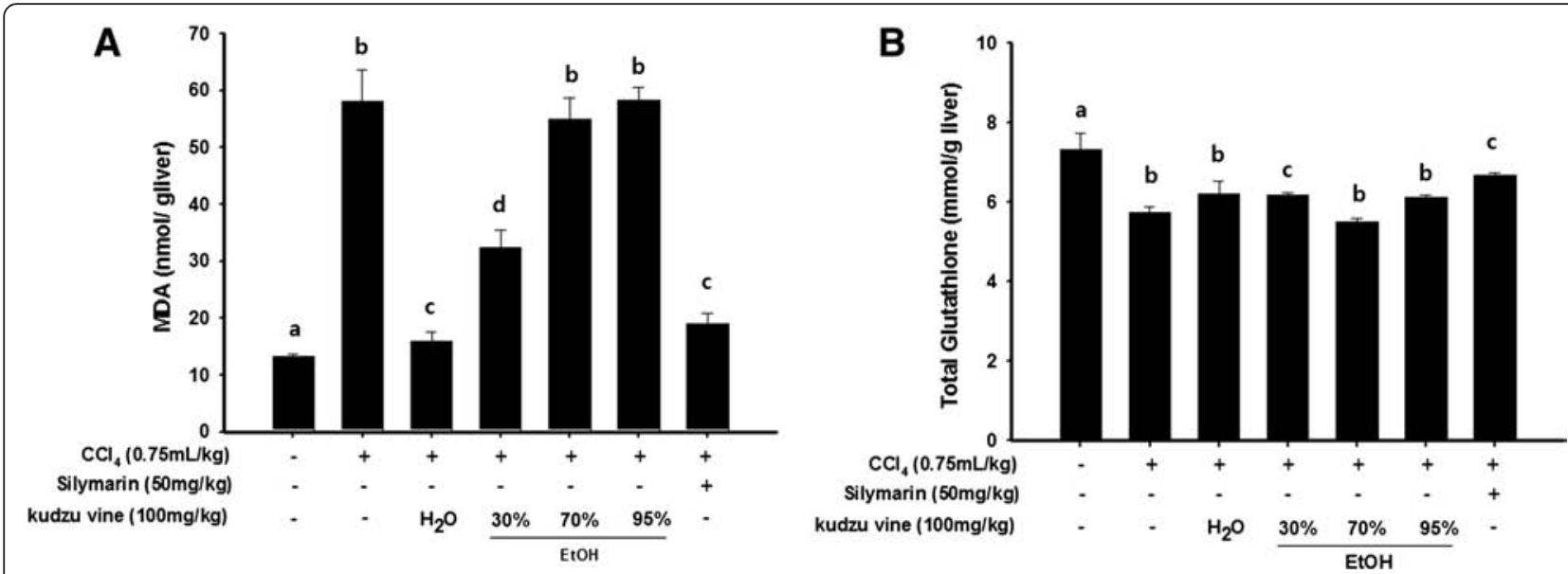

Fig. 5 Effects of kudzu vine extracts on hepatic (a) malondialdehyde and (b) total glutathione amount. The results are presented as mean \pm SD. Values with different letters $(a, b, c)$ are significantly different one from another (one-way ANOVA followed by Newman-Keuls multiple range test, $P<0.05)$

liver during the removal of xenobiotics and toxins, and oxidative stress caused by ROS has been linked to various liver diseases $[12,20,25]$. In comparison with normal cells, malignant cells seem to remain functional under higher levels of endogenous oxidative stress in vitro and in vivo [12, 26, 27].

We also used $\mathrm{CCl}_{4}$ to induce hepatotoxicity in rats. $\mathrm{CCl}_{4}$ is actively converted by cytochrome P-450, especially CYP2E1, in the liver tissues to its highly reactive trichloromethyl free radical $\mathrm{CCl}_{3}$. The $\mathrm{CCl}_{3}$ radical reacts with cellular macromolecular protein and polyunsaturated fatty acids $[12,13]$ in the presence of molecular oxygen, it forms more toxic trichloromethylperoxyl radicals $\mathrm{CCl}_{3} \mathrm{O}$ along with $\mathrm{H}_{2} \mathrm{O}_{2}, \mathrm{O}_{2}^{-}$, and $\mathrm{OH}$ $[28,29]$. So, $\mathrm{CCl}_{4}$ is conventionally used to induce liver toxicity, to allow the testing of drugs for their hepatoprotective property. The $\mathrm{CCl}_{4}$ treatment elevates the levels of plasma enzymes ALT and AST, which are indicators of cellular leakage and loss of functional integrity of cell membranes in the liver [30,31]. The activities of ALT and AST were significantly suppressed after the administration of kudzu vine extracts in the present study. This suggests that kudzu vine extract effectively protected hepatocytes against the toxic effects of $\mathrm{CCl}_{4}$, resulting in a reduction in plasma ALT and AST levels in $\mathrm{CCl}_{4}$-treated rats.

An increase in MDA levels, as seen in our study after $\mathrm{CCl}_{4}$ administration, indicates enhancement of lipid peroxidation, leading to tissue damage and failure of antioxidant defence mechanisms to prevent the formation of excessive free radicals [16, 28, 30]. Significant reductions in hepatic lipid peroxidation were detected only in the water and $30 \%$ kudzu vine ethanolic extract-treated groups. There were no significant changes observed in the $70 \%$ and $95 \%$ kudzu vine ethanolic extract-treated groups. Changes in MDA levels and plasma AST and ALT levels displayed the same trends.

GSH was reduced in the liver of $\mathrm{CCl}_{4}$-treated rats, while an increased GSH level was observed in conjunction with the administration of $30 \%$ ethanol-derived kudzu vine extract. GSH is a highly effective extra- and intra-cellular antioxidant compound that plays a central role in coordinating the body's antioxidant defence processes [26]. GSH protects cells against electrophilic attack from xenobiotics such as free radicals and peroxides [28, 29, 32].

Our results suggest that the potent radical scavenging activity of kudzu vine extracts ameliorates oxidative stress and terminates the chain reaction involved in lipid peroxidation. Therefore, the hepatoprotective effect of kudzu vine extract is mainly due to its ability to neutralize the increase of free radicals caused by chemicals. The potency of hepatoprotective activity in high concentration ethanolic extracts was lower than that of the low concentration ethanolic extracts in the rat model studied, indicating that the amount and kind of ingredients may vary depending on the extraction conditions. Further study into the underlying cause of these differences is needed.

\section{Conclusions}

Kudzu vine extracts mitigated the induction of oxidative stress, successfully restored liver function, and significantly alleviated chemical-induced hepatic damage. The kudzu vine might be useful in the prevention of oxidative damage in liver cells and tissues, and should be developed as a new natural drug for the treatment of liver injury by scavenging free radicals and boosting the endogenous antioxidant system. 


\section{Ethics approval and consent to participate} This study was approved by the Wonkwang University Animal Care Committee.

\section{Abbreviations}

ALT: alanine aminotransferase; AST: aspartate aminotransferase;

$\mathrm{CCl}_{4}$ : carbon tetrachloride; GSH: glutathione; HPLC: high-performance liquid chromatography; MDA: malondialdehyde; PBS: phosphate-buffered saline; ROS: reactive oxygen species; $t$-BHP: tertiary-butylhydroperoxide.

\section{Competing interests}

The authors declare that there are no conflicts of interests regarding the publication of this article.

\section{Authors' contributions}

All authors read and approved the final version of the manuscript. SYK, YCK, and HKC conceived and designed the experiments. BYC, DSL, and JKL performed the experiments. BYC, DSL, and JKL analyzed the data. SYK and BYC drafted the manuscript. SYK, YCK, HKC, BYC, DSL, and JKL revised the manuscript.

\section{Acknowledgements}

This paper was supported by Wonkwang University in 2015.

\section{Funding}

No specific funding was received for this study.

\section{Author details}

${ }^{1}$ Institute of Pharmaceutical Research and Development, College of Pharmacy, Wonkwang University, Shinyoung-dong 344-2, Iksan, Jeonbuk 570-749, Republic of Korea. ${ }^{2}$ Department of Biomedical Chemistry, College of Health and Biomedical Science, Konkuk University, Chung-Ju 27478, Republic of Korea. ${ }^{3}$ Han Poong Pharmaceutical Co., Ltd, 333-24 1st Palbok-dong, Deokjin-gu, Jeonju-si, Jeonbuk 561-841, Republic of Korea.

Received: 8 April 2015 Accepted: 27 January 2016

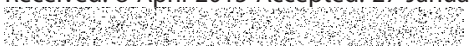

\section{References}

1. Yuan R, Lin Y. Traditional Chinese medicine: an approach to scientific proof and clinical validation. Pharmacol Ther. 2000;86(2):191-8.

2. Chen G, Li L. Nutrient consumption and production of isoflavones in bioreactor cultures of Pueraria lobata (Willd). J Environ Biol. 2007;28(2):321-6.

3. Cui SM, Zhao CS, He ZG. Assessment of Pueraria lobata isoflavone with selfmicroemulsifying drug delivery systems in vitro and in vivo. Zhong Yao Cai. 2007;30(6):684-7.

4. Xue C, Zhang J, Jing W, Wang Y, Si N, Liu A. Determination of five components in Pueraria lobata decoction with reference extraction method. Zhongguo Zhong Yao Za Zhi. 2012;37(16):2388-91.

5. Zou Y, Hong B, Fan L, Zhou L, Liu Y, Wu Q, et al. Protective effect of puerarin against beta-amyloid-induced oxidative stress in neuronal cultures from rat hippocampus: involvement of the GSK-3beta/Nrf2 signalling pathway. Free Radic Res. 2013;47(1):55-63.

6. Forseth IN, Innis AF. Kudzu (Pueraria montana): History, physiology, and ecology combine to make a major ecosystem threat. Crit Rev Plant Sci. 2004;23(5):401-13.

7. Tanaka T, Tang H, Yu F, Michihara S, Uzawa Y, Zaima N, et al. Kudzu (Pueraria lobata) vine ethanol extracts improve ovariectomy-induced bone loss in female mice. J Agric Food Chem. 2011;59(24):13230-7.

8. Chew MK. The monstering of tamarisk: how scientists made a plant into a problem. J Hist Biol. 2009;42(2):231-66.

9. Keung WM, Vallee BL. Kudzu root: an ancient Chinese source of modern antidipsotropic agents. Phytochemistry. 1998;47(4):499-506.

10. Landete JM. Dietary intake of natural antioxidants: vitamins and polyphenols. Crit Rev Food Sci Nutr. 2013;53(7):706-21.

11. Jaeschke H, McGill MR, Ramachandran A. Oxidant stress, mitochondria, and cell death mechanisms in drug-induced liver injury: lessons learned from acetaminophen hepatotoxicity. Drug Metab Rev. 2012;44(1):88-106.

12. Noh JR, Gang GT, Kim YH, Yang KJ, Hwang JH, Lee HS, et al. Antioxidant effects of the chestnut (Castanea crenata) inner shell extract in t-BHPtreated HepG2 cells, and $\mathrm{CCl}_{4}$ - and high-fat diet-treated mice. Food Chem Toxicol. 2010;48(11):3177-83.
13. Bell AN, Mehendale HM. The effect of dietary exposure to a mirex plus chlordecone combination on $\mathrm{CCl}_{4}$ hepatotoxicity. Toxicol Sci. 1985;5(4):679-87.

14. Klouwen HM. Determination of the sulfhydryl content of thymus and liver using DPPH. Arch Biochem Biophys. 1962:99:116-20.

15. Reitman S, Frankel S. A colorimetric method for the determination of serum glutamic oxalacetic and glutamic pyruvic transaminases. Am J Clin Pathol. 1957;28(1):56-63.

16. Draper $\mathrm{HH}$, Hadley M. Malondialdehyde determination as index of lipid peroxidation. Methods Enzymol. 1990;186:421-31.

17. Griffith OW, Meister A. Potent and specific inhibition of glutathione synthesis by buthionine sulfoximine (S-n-butyl homocysteine sulfoximine). J Biol Chem. 1979;254(16):7558-60.

18. Bengmark S. Curcumin, an atoxic antioxidant and natural NFkappaB, cyclooxygenase-2, lipooxygenase, and inducible nitric oxide synthase inhibitor: a shield against acute and chronic diseases. J Parenter Enteral Nutr. 2006;30(1):45-51.

19. Klaassen C. Casarett \& Doull's Toxicology: The Basic Science of Poisons, Eighth Edition (Casarett \& Doull's Toxicology). USA: McGraw-Hill Medical; 2013.

20. Arrigo T, Leonardi S, Cuppari C, Manti S, Lanzafame A, D'Angelo G, et al. Role of the diet as a link between oxidative stress and liver diseases. World J Gastroenterol. 2015;21(2):384-95.

21. Atmaca M, Bilgin HM, Obay BD, Diken H, Kelle M, Kale E. The hepatoprotective effect of coumarin and coumarin derivates on carbon tetrachlorideinduced hepatic injury by antioxidative activities in rats. J Physiol Biochem. 2011;67(4):569-76.

22. Li Y, Kong D, Ahmad A, Bao B, Sarkar FH. Antioxidant function of isoflavone and 3,3'-diindolylmethane: are they important for cancer prevention and therapy? Antioxid Redox Signal. 2013;19(2):139-50.

23. Hwang YP, Jeong HG. Mechanism of phytoestrogen puerarin-mediated cytoprotection following oxidative injury: estrogen receptor-dependent up-regulation of PI3K/Akt and HO-1. Toxicol Appl Pharmacol. 2008; 233(3):371-81.

24. Li L, Zhang CR. Production of puerarin and isoflavones in cell suspension cultures of Pueraria lobata (Willd): effects of medium supplementation with casein hydrolysate and coconut milk. J Environ Biol. 2006;27(1):21-6.

25. Linhart K, Bartsch H, Seitz HK. The role of reactive oxygen species (ROS) and cytochrome P-450 2E1 in the generation of carcinogenic etheno-DNA adducts. Redox Biol. 2014;3:56-62.

26. Senevirathne M, Ahn CB, Je JY. Hepatoprotective effect of chitooligosaccharides against tert-butylhydroperoxide-induced damage in Chang liver cells. Carbohydrate Polymers. 2011;83(2):995-1000.

27. Pareek A, Godavarthi A, Issarani R, Nagori BP. Antioxidant and hepatoprotective activity of Fagonia schweinfurthii (Hadidi) Hadidi extract in carbon tetrachloride induced hepatotoxicity in HepG2 cell line and rats. J Ethnopharmacol. 2013;150(3):973-81.

28. Rivera H, Shibayama M, Tsutsumi V, Perez-Alvarez V, Muriel P. Resveratrol and trimethylated resveratrol protect from acute liver damage induced by $\mathrm{CCl}_{4}$ in the rat. J Appl Toxicol. 2008;28(2):147-55.

29. Zhao HD, Zhang F, Shen G, Li YB, Li YH, Jing HR, et al. Sulforaphane protects liver injury induced by intestinal ischemia reperfusion through Nrf2-ARE pathway. World J Gastroenterol. 2010;16(24):3002-10.

30. Ganaie MA, Khan TH, Siddiqui NA, Ansari MN. Ameliorative effect of methanol extract of Rumex vesicarius on $\mathrm{CCl}_{4}$-induced liver damage in Wistar albino rats. Pharmaceut Biol. 2015;53(8):1163-7.

31. Khan MA, Gupta A, Sastry JL, Ahmad S. Hepatoprotective potential of kumaryasava and its concentrate against $\mathrm{CCl}_{4}$-induced hepatic toxicity in Wistar rats. J Pharm Bioallied Sci. 2015;7(4):297-9.

32. Gad AS, Khadrawy YA, El-Nekeety AA, Mohamed SR, Hassan NS, AbdelWahhab MA. Antioxidant activity and hepatoprotective effects of whey protein and Spirulina in rats. Nutrition. 2011;27(5):582-9. 